\title{
Análisis de la Investigación Cualitativa en el área de Biblioteconomía y Documentación (1981-2010)
}

\author{
Beatriz MORENA DE DiAGO \\ Doctora en Ciencias de la Documentación. Universidad Complutense de Madrid \\ bmorena.ic@gmail.com
}

Recibido: Enero 2013

Aceptado: Marzo 2013

\begin{abstract}
Resumen: Se presentan los resultados de un estudio de producción de la Investigación Cualitativa en el área de Biblioteconomía y Documentación. Se analiza las fuentes de publicación que concentran mayor número de contribuciones, los años e idiomas de estas contribuciones y la filiación institucional y geográfica de los autores de los artículos de investigación recuperados en las bases de datos Lisa (Library and Information Science Abstracts) y Lista (Library, Information Science \& Technology Abstracts), con especial énfasis en el análisis concreto del caso español. Se incluye una propuesta de sistematización de técnicas cualitativas de recogida de datos más recurrentes en la literatura consultada: técnicas conversacionales, técnicas documentales y técnicas observacionales.
\end{abstract}

Palabras clave: Investigación Cualitativa; Biblioteconomía y Documentación; Producción Científica; Recogida de Datos; Técnicas Cualitativas.

\section{Qualitative Research Analysis in the area of Library and Information Science: (1981-2010)}

\begin{abstract}
The results of a study of production of Qualitative Research in the area of Library and Information Science are presented. It analyses the sources of publications that concentrate more contributions, the years and the language of this contributions and the geographical and institutional affiliation of the authors of the research articles retrieved in the databases Lisa (Library and Information Science Abstracts) and Lista (Library, Information Science \& Technology Abstracts), with special emphasis on the particular analysis of the Spanish case. It includes a proposal for systematization of qualitative data collection techniques more recurrent in the literature: conversational techniques, documentation techniques and observational techniques.
\end{abstract}

Keywords: Qualitative Research; Library and Information Studies; Scientific Production; Data Collection; Qualitative Techniques. 


\section{INTRODUCCIÓN: LA INVESTIGACIÓN CUALITATIVA}

La Investigación Cualitativa es definida como el proceso de investigación que "obtiene datos del contexto en el que los fenómenos ocurren, en un intento de describirlos, como medio de determinar el proceso en que se enmarcan y las perspectivas de aquéllos que participan en ellos" (Gorman y Clayton, 2005: 3).

La Investigación Cualitativa implica utilizar una metodología cualitativa para identificar de una manera más profunda a los individuos estudiados y conocer la razón por la que un determinado fenómeno ocurre en un contexto concreto. De ahí que su utilización englobe cuestiones de eficiencia, personalización, calidad y satisfacción del usuario. Cuando se contextualiza la Investigación Cualitativa en el área de Biblioteconomía y Documentación los profesionales del ámbito deben explorar las emociones, experiencias, situaciones y actitudes humanas que sus usuarios experimentan con la utilización de los productos y servicios de información para evaluarlos y mejorar su calidad permitiendo, a su vez, poner en funcionamiento otros nuevos.

En esencia un investigador cualitativo no busca "la verdad" o "la moralidad" sino una comprensión detallada de las perspectivas de otras personas enmarcadas en múltiples realidades mediante una metodología cualitativa no tan refinada y estandarizada como otros enfoques de investigación.

Delgado López-Cózar (2001) destaca la amplia variedad de metodologías empleadas en Biblioteconomía y Documentación ofreciendo un diagnóstico general de los métodos científicos durante el período 1965-1985. En el caso de los métodos cualitativos los porcentajes de uso son escasos, entre el 0'7\% en 1965 al 1' $6 \%$ en 1985 . Pero aboga que para la constitución de una auténtica disciplina "los métodos cualitativos pueden y deben jugar un significativo papel en la consecución de la ansiada meta" (Delgado López-Cózar, 2002: 150).

La tendencia actual muestra el predominio de uso de metodología cualitativa en la literatura especializada del área. En la siguiente Tabla 1 se recoge el número total de ítems proporcionados por las bases de datos Lisa y Lista para los términos de búsque$\mathrm{da}^{1}$ "quantitative research" y "qualitative research". Será el auge de la recuperación de la información en sistemas automatizados una de las razones de su incorporación en el ámbito debido a su necesaria adaptación a las necesidades de los usuarios.

"En el futuro, los métodos para estudiar las necesidades de los usuarios surgirán, cada vez más, del mundo cualitativo y menos del cuantitativo" (Frías y Borrego, 2004: 194).

${ }^{1}$ Fecha de consulta: 11-01-2013. (Campo AB- resumen). 
Tabla 1 Número de ítems recuperados en las bases de datos Lisa y Lista. Fuente: elaboración propia

\begin{tabular}{|l|c|c|}
\hline & $\begin{array}{c}\text { N. }{ }^{\circ} \text { ítems de la base de } \\
\text { datos Lisa }\end{array}$ & $\begin{array}{c}\text { N. }{ }^{\circ} \text { ítems de la base de } \\
\text { datos Lista }\end{array}$ \\
\hline "Qualitative research" & 357 & 368 \\
\hline "Quantitative research" & 111 & 94 \\
\hline
\end{tabular}

Por otro lado, Booth (2009) presenta los resultados de un análisis de contenido de trabajos del área de Biblioteconomía y Documentación publicados entre los años 2007-2008 para determinar las características de la Investigación Cualitativa. Entre las conclusiones alcanzadas destaca que el 3\% de la Investigación Cualitativa ocurre en el contexto de estudios que emplean métodos mixtos. El término métodos mixtos se utiliza para la combinación de métodos cuantitativos y cualitativos en una misma investigación (Fidel, 2008). En estos casos, lo más importante es que la elección de métodos cuantitativos y/o cualitativos se haga en términos de obtención de mejores respuestas al problema de investigación.

Las técnicas cualitativas de recogida de datos forman parte del dominio metodológico del proceder cualitativo siendo la base del trabajo de campo. Se trata de unos instrumentos que permiten al investigador cualitativo interactuar con los individuos o con los grupos definidos existiendo una relación horizontal no jerárquica entre el investigador y el sujeto de estudio.

En ocasiones, los métodos y las técnicas de recogida de datos son tratados por los autores de manera conjunta bajo el epígrafe métodos de investigación cualitativa constatándose la falta de diferenciación métodos/técnicas cualitativas motivada por la ausencia de una única clasificación de técnicas cualitativas y la dispersión de la aplicación de la Investigación Cualitativa en investigaciones de diversas ramas (Borrego Huerta, 2001: 17). Con la dificultad añadida de que en muchos casos se listan distintas técnicas de recogida de datos que no se integran en la temática cualitativa, debido a que tienen sobrada entidad como en el caso de las entrevistas, los focus groups o la observación participante.

Se ha constatado que si se quiere recuperar la literatura existente sobre la aplicación y/o análisis de una/s determinada/s técnica/s cualitativa/s debemos fijar la estrategia de búsqueda a partir de esa/s técnica/s específica/s. Pero si se quiere estudiar el conjunto de técnicas cualitativas de recogida de datos utilizadas en el área de Biblioteconomía y Documentación el término de búsqueda debe ser global con el término canónico "Investigación Cualitativa". 


\subsection{TÉCNICAS CUALITATIVAS DE RECOGIDA DE DATOS EN EL ÁREA DE BIBLIOTECONOMÍA Y DOCUMENTACIÓN}

Las técnicas cualitativas no han recibido tradicionalmente una excesiva atención en las monografías y manuales en nuestra disciplina. Fue en 1990, cuando Mellon publica el primer manual sobre la aplicación de métodos cualitativos en Biblioteconomía y Documentación (Borrego Huerta, 2001: 22). Sin embargo, en la literatura tenemos ejemplos de la aplicación temprana de estas técnicas como el estudio de Bruyn (1970) que utiliza la técnica de la observación participante en el área o la técnica del incidente crítico empleada en algunas bibliotecas en las décadas de los años 60 y 70 (Balboa, 2008), etc. De hecho, es preciso señalar que una gran mayoría de trabajos consultados aplican técnicas cualitativas de recogida de datos pero no versan sobre Investigación Cualitativa como tema/objeto de estudio.

Borrego Huerta (2001) analiza, clasifica y contabiliza las técnicas cualitativas de recogida de datos en artículos empíricos del área utilizando la clasificación de Patton (1990) en torno a tres grandes familias de técnicas de recogida de datos cualitativos (entrevistas, las técnicas de observación y los documentos). En función de los datos absolutos ofrecidos de cada técnica concluye que las entrevistas en profundidad, los documentos y la observación son las técnicas cualitativas más empleadas en este orden, respectivamente. Años más tarde, Booth (2009), a pesar de no aportar una sistematización concreta, indica que las entrevistas, los cuestionarios ${ }^{2}$ y las observaciones son las técnicas más comunes en investigaciones cualitativas en Biblioteconomía y Documentación y la técnica Delphi y los focus groups, aunque menos habituales, también aparecían representadas en la literatura especializada.

En un intento de clasificación, Frías Montoya (2010: 42) agrupa las técnicas cualitativas de recogida de datos en tres tipos: las técnicas conversacionales, las técnicas observacionales y las técnicas documentales. Dentro de las llamadas técnicas conversacionales se centra en la entrevista cualitativa, en los grupos de discusión y otras técnicas afines como la técnica Delphi y la investigación cooperativa. Con respecto a las llamadas técnicas observacionales, diferencia cuatro tipos de observación cualitativa: panorámica-participante, panorámica no participante, selectiva-participante y selectiva-no participante.

Entre los manuales sobre Investigación Cualitativa aplicados al área de Biblioteconomía y Documentación destacan las obras de Powell y Connaway (2004), Gorman y Clayton (2005), Beck y Manuel (2008), Lawal (2009) o Goodman (2011), entre otros.

\footnotetext{
${ }^{2}$ Murua Anzola (2007: 36) señala que el cuestionario es una técnica de investigación cualitativa aunque no la más representativa, planteando el término cuestionario mixto como aquel que recoge preguntas abiertas y cerradas.
} 
Powell y Connaway (2004) resaltan la primacía de cinco técnicas de recolección de datos: el cuestionario, la entrevista, los grupos de discusión, la entrevista telefónica y la observación. Gorman y Clayton (2005) distinguen cuatro técnicas cualitativas de recogida de datos: la observación, la entrevista, los focus groups y la investigación histórica, ésta última, entendida como investigación documental (incluyendo los testimonios orales generalmente grabados o transcritos). En cuanto a la observación, diferencian dos tipos: la observación participante o estructurada y la observación no intrusiva (no estructurada). En la entrevista distinguen dos tipos básicos, las entrevistas estructuradas o survey interviews donde las preguntas y respuestas están predeterminadas y las entrevistas no estructuradas, también llamadas entrevistas intensivas o en profundidad, donde la formulación exacta de las preguntas ni de las respuestas ha sido predeterminada. En relación con la técnica del focus group, prestan especial atención a las técnicas storytelling y al grupo nominal, en ocasiones también nombrado "tormenta de ideas de calidad" (quality brainstorm).

Beck y Manuel (2008) en su manual sobre métodos de investigación para bibliotecarios y profesionales de la información destacan las entrevistas y los focus groups, la observación y los estudios de usabilidad, la investigación-acción y la investigación en el aula o classroom research (aunque ambas están englobadas en la investigaciónacción, difieren en que el centro de interés en la investigación-acción está en solventar un problema mientras que en la investigación en el aula no existe ningún problema), la investigación experimental (definida casi siempre como cuantitativa), la bibliometría también cuantitativa y el análisis de contenido.

Por su parte, Lawal (2009) condensa métodos y técnicas de investigación cualitativa indistintamente, como la observación, la entrevista y el grupo de discusión, incluyendo otros métodos de diseño no experimental ${ }^{3}$ como la investigación histórica y los estudios de caso. Finalmente, Goodman (2011) menciona que hay muchas técnicas para recoger datos durante una investigación cualitativa. En su caso, seleccionando los datos que pueden ser más útiles en el área de Biblioteconomía y Documentación, recoge la observación participante, los focus groups, las entrevistas, los estudios de caso, los métodos no intrusivos (que no requieren la participación activa del sujeto) y la exploración de historias y narraciones.

Hemos fijado una propuesta de sistematización con los tres grandes grupos/familias de técnicas cualitativas de recolección de datos más recurrente en la literatura especializada, con la intención de contribuir a la reducción de las clasificaciones existentes (recogida en el Anexo: Propuesta de sistematización):

3 "Los métodos de investigación no experimental exploran las preguntas de investigación sin manipulación de una variable" (Lawal, 2009: 71). 
1) Técnicas conversacionales: su función básica consiste en generar una conversación con los usuarios de forma activa utilizando la entrevista cualitativa ${ }^{4}$, el focus group ${ }^{5}$ y otras técnicas afines como la técnica Delphi, el incidente crítico $^{6}$, etc.

2) Técnicas documentales o investigación documental. Emplean documentos públicos y/o privados que mediante el análisis de contenido, pragmático y/o del discurso son utilizados para los fines de la investigación. Se aplica a todo tipo de documentos (textuales, audiovisuales, numéricos, etc.).

3) Técnicas observacionales: se centran en la atención sistemática a un objetivo determinado con el fin de obtener información. Se han señalado los protocolos verbales ${ }^{7}$ y la técnica de la observación. Además, hemos recogido un parámetro de clasificación de la observación (la más recurrente en la literatura consultada) en función del grado de participación del observador.

A continuación, analizaremos el paradigma cualitativo en las dos subáreas más productivas del área de Biblioteconomía y Documentación: la recuperación de información y los estudios de usuarios (Fernández Hernández y Rivera, 2009: 13).

\subsection{LA RECUPERACIÓN DE INFORMACIÓN}

Para Ruiz Abellán y López-Huertas Pérez (1999) la aproximación a la representación conceptual de los usuarios de sistemas de información utilizando la perspectiva cualitativa se realiza a través de dos grupos de técnicas: las técnicas directas y las técnicas indirectas. Estos grupos se establecen en función del grado de participación del usuario en la investigación. De tal manera que, en las técnicas directas el usuario está implicado en la investigación y en las técnicas indirectas se analiza el contexto del usuario sin que éste sea consciente de su participación. Las principales técnicas

\footnotetext{
${ }^{4}$ La entrevista es la conversación mantenida con el usuario con el propósito de obtener información sobre un asunto determinado.

${ }^{5}$ El focus group, también llamado por algunos autores grupos de discusión, consiste en una reunión de personas que debaten sobre un tema para que a partir de esa interacción surjan puntos de consenso, opiniones y sentimientos, nuevas ideas, etc.

${ }^{6}$ La técnica del incidente sirve para recoger datos sobre los comportamientos de los usuarios inmediatamente después que se haya realizado ese incidente o actividad humana observable.

7 Según Borrego Huerta (2001: 152) los protocolos verbales también se llaman "think aloud" forman parte de las técnicas de observación. Aunque, Matthews (2007) considera que son una variante de la entrevista, porque sirve para que los usuarios verbalicen sus pensamientos mientras realizan la actividad. Hemos mantenido la subdivisión de Borrego Huerta por el componente clave de los protocolos con respecto a la observación del comportamiento.
} 
indirectas en el campo de la lingüística documental son tres: el análisis de contenido ${ }^{8}$, el análisis del discurso y el análisis pragmático que concreta las posibles lecturas que los usuarios pueden dar al texto. En el grupo de las técnicas directas destacan las entrevistas/cuestionarios, las fichas conceptuales, las dinámicas psico-grupales y las técnicas proyectivas que "permiten conocer aspectos inconscientes de las actitudes de los usuarios" (Ruiz Abellán y López-Huertas Pérez, 1999: 440). En el tipo entrevistas/cuestionarios se engloba la técnica Delphi porque utiliza un cuestionario estructurado entre un grupo de expertos (sin que interactúen entre ellos, de manera anónima) para obtener finalmente un consenso.

Esta clasificación nos aporta la posibilidad de vincular estas técnicas a dos tipologías de usuarios: usuarios activos (los que conocen el objetivo de la investigación formando parte activa en la misma como fuente de datos de manera presencial o virtual) y usuarios pasivos (usuarios que desconocen su participación en la investigación aunque están implicados en ella). Además, en ambos casos los usuarios pueden ser internos o externos a las unidades de información y documentación.

Tabla 2. Clasificación de usuarios en función de las técnicas cualitativas de recogida de datos. Fuente: elaboración propia

\begin{tabular}{|l|l|l|}
\hline TÉCNICAS DIRECTAS & $\Rightarrow$ & USUARIOS ACTIVOS (reales $^{9}$ y potenciales $^{10}$ ) \\
\hline TÉCNICAS INDIRECTAS & $\Rightarrow$ & USUARIOS PASIVOS (reales y potenciales) \\
\hline
\end{tabular}

Pérez-Montoro Gutiérrez (2010: 337) menciona los "métodos participativos aplicados en entornos web" (también llamados "metodología denominada persona") cuando se refiere a una de las fases de análisis de la arquitectura de la información denominada análisis de usuarios. Estos métodos estudian los comportamientos de los usuarios en la web. Las principales estrategias metodológicas participativas son los cuestionarios, los grupos de discusión o focus groups, las entrevistas, el card sorting ${ }^{11}$, la técnica del eyetrack $^{12}$ o los test con usuarios ${ }^{13}$.

8 "El objetivo es estudiar los textos o productos documentales que contextualizan el mundo informativo-documental del usuario [...] para poder crear mapas conceptuales” (Ruiz Abellán y López-Huertas Pérez, 1999: 436).

${ }^{9}$ Usuarios reales "son aquellos que no sólo son conscientes que necesitan la información sino que la utilizan frecuentemente" (Sanz Casado, 1994: 19).

${ }^{10}$ Usuarios potenciales "aquéllos que necesitan información para el desarrollo de sus actividades, pero no son conscientes de ello, por lo tanto, no expresan sus necesidades" (Sanz Casado, 1994: 19).

11 "Originariamente, esta técnica fue diseñada por los psicólogos como método para estudiar cómo las personas organizan y categorizan su propio conocimiento. En los entornos web y en un sentido general, consiste en una técnica en la que se pide a un pequeño grupo de potenciales usuarios de la web que realice una agrupación de tarjetas (etiquetas o conceptos) que refleje los modelos mentales de esos mismos usuarios y que sirva también de inspiración para que los diseñadores puedan estructurar un sitio web a partir de esos modelos”. (Pérez-Montoro Gutiérrez, 2010: 340). 
En el Informe APEI sobre usabilidad, Hassan Montero y Ortega Santamaría (2009: 43) recogieron las metodologías y técnicas del "Diseño Centrado en el Usuario" (DCU) en la planificación de arquitecturas de información, ampliando la tipología plasmada por Pérez-Montoro Gutiérrez (2010). Se trataba del test de usuarios (técnica principal para evaluar la usabilidad de un diseño), la evaluación heurística, el card sorting o agrupación de tarjetas, eye-tracking o técnica de seguimiento visual, la etnografía y otras técnicas destacables como las entrevistas, encuestas y la analítica web (referida al análisis de datos reales de uso del sitio web).

Tabla 3. Principales técnicas aplicadas al Diseño Centrado en el Usuario. Fuente: Hassan Montero y Ortega Santamaría (2009: 43-52).

\begin{tabular}{|c|c|c|c|}
\hline Técnicas & $\begin{array}{c}\mathrm{N}^{0} \text { de participan- } \\
\text { tes }\end{array}$ & Función & Limitaciones \\
\hline Test de usuarios & 15 & $\begin{array}{l}\text { Conocer la primera impresión del } \\
\text { usuario, los errores cometidos por } \\
\text { los actores, el tiempo empleado y } \\
\text { la satisfacción final. }\end{array}$ & $\begin{array}{c}\text { Alto coste } \\
\text { Interacción con el } \\
\text { usuario descontextuali- } \\
\text { zada }\end{array}$ \\
\hline $\begin{array}{c}\text { Evaluación heurís- } \\
\text { tica }\end{array}$ & 3-5 expertos & $\begin{array}{l}\text { Conocer los problemas de usabili- } \\
\text { dad en relación con unos principios } \\
\text { previamente establecidos (princi- } \\
\text { pios heurísticos) }\end{array}$ & $\begin{array}{c}\text { Menos eficaz en la } \\
\text { detección de problemas } \\
\text { de usabilidad que ten- } \\
\text { drán mayor impacto en } \\
\text { los usuarios }\end{array}$ \\
\hline Card sorting & 5 & $\begin{array}{l}\text { Identificar la relación semántica y } \\
\text { el grado de relación de los concep- } \\
\text { tos representados en cada tarjeta }\end{array}$ & $\begin{array}{l}\text { Su uso inexper- } \\
\text { to/inadecuado genera } \\
\text { resultados erróneos }\end{array}$ \\
\hline Eye-tracking & Sin especificar & $\begin{array}{l}\text { Predecir el comportamiento visual } \\
\text { del usuario, resaltando los elemen- } \\
\text { tos que atraerán su atención }\end{array}$ & $\begin{array}{l}\text { No todos los ojos pue- } \\
\text { den calibrarse. } \\
\text { Tecnología cara }\end{array}$ \\
\hline
\end{tabular}

Según González Teruel (2005: 133) las técnicas de recogida de información más comúnmente utilizadas para investigar las necesidades y usos de la información son la observación, la encuesta (cuestionario, entrevistas, técnica del incidente crítico y focus group), las técnicas de consenso (en concreto la técnica Delphi), el análisis de citas y referencias y el análisis de las transacciones entre usuario y sistema.

Existen diferentes clasificaciones de card sorting: abierto y cerrado, individual y colectivo, presencial y virtual.

12 "Herramienta informática que permite registrar el movimiento de los ojos de un usuario cuando visita una página web (o mira una pantalla de ordenador, en definitiva) e identificar las zonas de esas páginas sobre las que se detiene a visionar" (Pérez-Montoro Gutiérrez, 2010: 347).

13 "La técnica consiste en poner a prueba con diversos grupos de usuarios la arquitectura de la página existente o una propuesta arquitectónica que ulteriormente se quisiera implementar, recogiendo para ello las opiniones de esos usuarios cuando se encuentran realizando diversas tareas en la página analizada" (Pérez-Montoro Gutiérrez, 2010: 351-352). 


\subsection{LOS ESTUDIOS DE USUARIOS}

Hernández Salazar (2008: 14) en un estudio de producción determina que los métodos y/o técnicas cualitativas para realizar estudios de usuarios más habituales son el análisis de contenido, el análisis de zonas de intervención, las dinámicas de grupos, el estudio de caso, los focus groups, el incidente crítico, el materialismo dialéctico y la observación participante.

Sin embargo, unos años más tarde, Hernández Salazar (2011: 357-358) resumía las principales técnicas cualitativas en el contexto de los estudios de satisfacción dentro de las actividades de información en: el incidente crítico, el grupo focal, el análisis de contenido, el debate en grupos de interés, la simulación de uso y la gestión de reclamaciones. Distinguiéndolas de lo que identifica como "instrumentos de recogida de datos" que eran los cuestionarios, entrevistas, ensayos, documentos (por ejemplo, diarios, cuadernos de campo, fotografías) y registros grabados (audio, video y audiovisuales).

Con respecto a la simulación de uso, los investigadores (sin identificarse de esta manera) solicitan un recurso o servicio para conocer si se está ofreciendo al usuario de manera adecuada. De tal manera que, permite medir de forma minuciosa la calidad del servicio y la satisfacción del usuario. Esta técnica, también referenciada como "Cliente oculto", "pseudocompras" o "Mystery Shopping" fue utilizada por primera vez en España por Riaza Chaparro (2007: 56) en la Red de bibliotecas de Obra Social Caja Madrid. Por lo que Matthews (2007: 49) nos recuerda que la fortaleza de las técnicas cualitativas se clasifica en tres grandes grupos en función del contacto con el usuario.

Tabla 4. Tipología de técnicas cualitativas de Matthews (2007: 49)

\begin{tabular}{|l|l|l|}
\hline \multicolumn{1}{|c|}{ Sin contacto } & \multicolumn{1}{|c|}{ Contacto uno a uno } & \multicolumn{1}{c|}{ Interacción de grupo } \\
\hline - Examen de documentos & - Observación & - Focus group \\
- Diarios & -Entrevista & - Método Delphi \\
& -Teoría inductiva (Grounded theory) & - Técnica del incidente crítico \\
& - Protocolos verbales & - Mapas conceptuales \\
& - Métodos etnográficos & \\
\hline
\end{tabular}

Por su parte, Sanz Casado (1994) agrupa "los métodos" más utilizados para recoger datos sobre hábitos y necesidades de información de los usuarios en dos tipos:

\footnotetext{
14 "En algunos casos el análisis de texto es llamado hermenéutica, la cual se refiere al desarrollo y estudio de teorías de la interpretación y comprensión de textos" (Matthews, 2007: 50).

${ }^{15}$ Dentro de estos métodos indica que las dos técnicas principales son la observación participante y la entrevista (Matthews, 2007: 54).

${ }^{16}$ Permiten representar el conocimiento en grafos. "El proceso proporciona una metodología para organizar ideas de un grupo o crear un marco de trabajo común que pueda ser usado para la planificación y la evaluación” de una biblioteca, por ejemplo (Matthews, 2007:56).
} 
1. Métodos directos "cuando se pretende conseguir que sea el propio usuario quién defina sus hábitos, necesidades o el uso que hace de la información que solicita" (Sanz Casado, 1994: 91). Dentro de esta tipología destaca el método Delphi, las encuestas por correo (indicando la doble tipología de preguntas abiertas o cerradas que pueden contener los cuestionarios), la entrevista personal y la técnica del incidente crítico (incluida dentro de la entrevista personal).

2. Métodos indirectos "permite[n] conocer de forma indirecta, sin tener que consultar a los usuarios, sus hábitos y necesidades de información" (Sanz Casado, 1994: 101). Los cuatro tipos indirectos señalados son el análisis de citas, el análisis de referencias, el análisis de las peticiones de documentos y la observación. Ésta puede llevarse a cabo de forma estructurada o de forma no estructurada, es decir, sin un plan previo para registrar los comportamientos de los usuarios en la búsqueda y uso de la información.

También, Martín Moreno (2007: 135) diferencia entre métodos directos y métodos indirectos como los dos grandes grupos (en función de la actuación del usuario) para recoger información sobre los mismos. Dentro de los métodos directos señala las tres técnicas más utilizadas en trabajos que versan sobre hábitos y necesidades de información de los usuarios. Se trata de la encuesta auto-administrada, la entrevista que considera variante de la encuesta porque "consiste en una conversación en la que el entrevistador formula preguntas, normalmente preparadas previamente y a ser posible encuadradas en un cuestionario" (Martín Moreno, 2007: 138) y el incidente crítico (considerada una variante de la entrevista personal). Dentro de la técnica directa de la entrevista diferencia dos tipos: personal y telefónica en función de la presencia del usuario. Sin embargo, tan sólo destaca dos técnicas indirectas como el análisis de referencias y el análisis de peticiones de documentos. Es decir que, los datos se extraen de documentos que han sido generados con un propósito distinto al propio estudio de usuarios.

\section{METODOLOGÍA}

El estudio de producción está basado en 521 trabajos-fuente procedentes de las bases de datos Lisa y Lista, con una cobertura temporal definida entre los años 1981 y 2010.

La elección de estas dos bases de datos está fundamentada en tres criterios: son bases de datos en línea especializadas exclusivamente en el área de Biblioteconomía y Documentación, con cobertura internacional y de obligada referencia para investigadores/docentes españoles del área.

En cualquier caso el análisis de las contribuciones aparecidas en ambas bases de datos nos permitirá caracterizar el análisis de producción de la Investigación Cualita- 
tiva aplicadas en el diseño y desarrollo de unidades de información y documentación en el ámbito internacional.

En nuestro caso la selección de los términos de búsqueda se basó en el análisis del estado de la cuestión de la literatura especializada. Por derivación acudimos a diferentes tesauros externos a las propias bases de datos utilizadas para el análisis de producción con el fin de contrastar la validez de los operandos de búsqueda seleccionados.

Aquí encontramos la principal dificultad que ha presentado el trabajo. Como señala Borrego Huerta (2001: 17) existe una confusión terminológica por el "número desconcertante de términos que han sido empleados para hacer referencia a la investigación cualitativa".

En consecuencia, a modo de síntesis, el operando/término de búsqueda utilizado para analizar la producción de trabajos de Investigación Cualitativa en el área de Biblioteconomía y Documentación fue "qualitative research" por ser, hoy por hoy, el más generalizado.

Finalmente, determinamos que el campo por el cual analizaríamos la producción existente sería el campo Resumen (AB), ya que, el número de resultados entre ambas bases de datos era bastante equitativo (en concreto la base de datos Lista proporcionaba 268 registros y la base de datos Lisa proporcionaba 253 registros) lo que, en última instancia, permitirá una comparación posterior de ambos resultados.

El enfoque cuantitativo del trabajo conlleva el uso y aplicación de indicadores bibliométricos a la literatura científica proporcionada por ambas bases de datos. En nuestro caso, emplearemos distintos indicadores de producción que aplicaremos sobre los resultados obtenidos de la base de datos Lisa y de la base de datos Lista para analizar las fuentes de publicación que concentran mayor número de trabajos, los años e idiomas de las contribuciones que versan sobre el tema/objeto de nuestro trabajo y la filiación institucional y geográfica de los autores.

\section{RESULTADOS}

\subsection{FUENTES DE PUBLICACIÓN QUE CONCENTRAN UN MAYOR NÚMERO DE CONTRIBUCIONES ANALIZADAS}

El número total de fuentes de publicación recogidas de los resultados de la base de datos Lisa suman un total de $124 \mathrm{y}$ en la base de datos Lista asciende a 122.

Tabla 5. Distribución de fuentes de publicación en función del número de ítems asociados a un mismo título. Fuente: elaboración propia.

\begin{tabular}{|c|c|c|}
\hline N. $^{\circ}$ de contribuciones & $\begin{array}{c}\text { N. }^{\circ} \text { de publicaciones de la base } \\
\text { de datos Lisa }\end{array}$ & $\begin{array}{c}\text { N. }{ }^{\circ} \text { de publicaciones de la } \\
\text { base de datos Lista }\end{array}$ \\
\hline Con 1 & 76 & 77 \\
\hline Con 2 & 20 & 17 \\
\hline Con 3 & 7 & 6 \\
\hline Con 4 & 6 & 10 \\
\hline
\end{tabular}




\begin{tabular}{|c|c|c|}
\hline Con 5 & 6 & 1 \\
\hline Con 6 & 4 & 4 \\
\hline Con 7 & 4 & 1 \\
\hline Con 8 & 1 & 3 \\
\hline Con 9 & -- & 1 \\
\hline Con 10 & -- & -- \\
\hline Con 11 & -- & 1 \\
\hline Con 12 & -- & -- \\
\hline Con 13 & -- & -- \\
\hline Con 14 & -- & 1 \\
\hline
\end{tabular}

Los títulos más productivos en función del número de ítems asociados a una fuente de publicación se clasificaron junto a la secuencia numérica (1-3) referida a los niveles de posicionamiento de los títulos concretos por el número total de ítems adscritos a dichas fuentes de publicación.

Tabla 6. Clasificación de fuentes de publicación más productivas de los resultados de las bases de datos Lisa y Lista. Fuente: elaboración propia

\begin{tabular}{|l|l|c|}
\hline \multicolumn{2}{|c|}{ Resultados de la base de datos Lisa } \\
\hline Clasificación & \multicolumn{1}{|c|}{ Fuentes de publicación } & $\frac{\mathrm{N}^{\mathbf{0}} \text {. total de }}{\text { items }^{17}}$ \\
\hline 1 & Library and Information Science Research & 8 \\
\hline 2 & Aslib Proceedings: New Information Perspectives & 7 \\
\hline 2 & Journal of Documentation & 7 \\
\hline 2 & Library Quarterly & 7 \\
\hline 2 & Library Trends & 7 \\
\hline 3 & Education for Information & 6 \\
\hline 3 & Evidence Based Library and Information Practice & 6 \\
\hline 3 & Journal of the Medical Library Association (JMLA) & 6 \\
\hline 3 & Social Science Computer Review & 14 \\
\hline \multicolumn{1}{|c|}{ Resultados de la base de datos Lista } \\
\hline 1 & Choice: Current Reviews for Academic Libraries & 11 \\
\hline 2 & Journal of Academic Librarianship & 9 \\
\hline 3 & Evidence Based Library \& Information Practice \\
\hline
\end{tabular}

Entre el cómputo global de fuentes de publicación de los resultados de ambas bases de datos, aunque no entre las más productivas, destacan los títulos de origen español El Profesional de la Información y Revista Española de Documentación Científi$c a$ (ésta sólo tiene presencia en los resultados de la base de datos Lisa).

${ }^{17}$ Cómputo total de trabajos que pertenecen a cada fuente de publicación. 


\subsection{AÑOS DE PUBLICACIÓN E IDIOMAS DE LAS CONTRIBUCIONES ANALIZADAS}

La tendencia general en los resultados de ambas bases de datos es el crecimiento continuado coincidiendo también en el año 2006 como el año más productivo con un número muy similar de contribuciones recogidas en ambas bases de datos (treinta y tres contribuciones entre los resultados de la base de datos Lisa y treinta y dos en la base de datos Lista). Con respecto al segundo año más productivo existen discrepancias entre los resultados. En la base de datos Lisa se posiciona en segundo lugar el año 2009 con un total de veinticinco contribuciones. Mientras que, en los resultados de la base de datos Lista comparten segunda posición los años 2008 y 2007 con un total de veintinueve contribuciones, respectivamente.

El período temporal comprendido entre los años 2006-2010 se configura como el más productivo, suponiendo el 43,5\% del total de contribuciones recuperadas en Lisa, y el 50\% de los resultados de la base de datos Lista.

Por otro lado, los resultados de ambas bases de datos muestran la utilización mayoritaria de la lengua inglesa en función de su proyección internacional. En el caso de los registros de la base de datos Lisa aparecen trece lenguas distintas al inglés en 33 ítems frente a los seis idiomas resultantes en 14 ítems de la base de datos Lista.

No existe coincidencia en la lengua no inglesa más utilizada entre las contribuciones sobre nuestro tema de estudio. En la base de datos Lisa ha sido el chino con un total de nueve contribuciones y en la base de datos Lista la lengua portuguesa con seis ítems. El punto común se sitúa en que, en ambos casos, el idioma español se configura como la segunda lengua no inglesa más productiva en la materia estudiada.

\subsection{PROCEDENCIA INSTITUCIONAL DE LOS AUTORES DE LAS CON- TRIBUCIONES ANALIZADAS}

Para conocer las instituciones más productivas en relación con nuestro tema/objeto de investigación, los datos de filiación institucional de los autores de las contribuciones se han agrupado en torno a una clasificación previa de tres niveles: universidades y otros centros de enseñanza, entidades científicas no universitarias y un último nivel que agrupa bibliotecas, centros de documentación, archivos y museos públicos y privados. Además se ha consignado una única procedencia institucional para aquellos autores con más de una contribución firmada. En estos casos las procedencias institucionales más actuales o las últimas consignadas según el año de publicación de las contribuciones.

El resultado final será una relación de aquellas instituciones más productivas que cuentan con investigadores especializados en la materia objeto de estudio en torno a la secuencia numérica (1-3) relacionando producción/filiación. 
Tabla 7. Instituciones más productivas según los resultados de las bases de datos Lisa y Lista. Fuente: elaboración propia.

\begin{tabular}{|l|l|}
\hline \multicolumn{2}{|c|}{ Resultados de la base de datos Lisa } \\
\hline 1 & The University of Sheffield \\
\hline 2 & University College London \\
\hline 3 & Manchester Metropolitan University \\
\hline 3 & Monash University \\
\hline 3 & Robert Gordon University \\
\hline 3 & Universidad de Barcelona \\
\hline 3 & University of Illinois at Urbana-Champaign \\
\hline \multicolumn{2}{|c|}{ Resultados de la base de datos Lista } \\
\hline 1 & The University of Sheffield \\
\hline 2 & University College London \\
\hline 2 & University of Pretoria \\
\hline 3 & Indiana University \\
\hline 3 & Kent State University \\
\hline 3 & Texas A\&M University \\
\hline 3 & University of Maryland \\
\hline 3 & University of Washington \\
\hline
\end{tabular}

De la lista anterior observamos que las instituciones resultantes forman parte del primer tipo de clasificación presentado "universidades y otros centros de enseñanza". Dentro de los resultados expuestos de la base de datos Lista no se recoge ninguna institución de origen español. Sin embargo, en la relación completa de filiaciones institucionales de los autores de las contribuciones recuperadas en esta base de datos se recogen cuatro instituciones de enseñanza superior de origen español apreciándose la situación predominante de las universidades catalanas.

En la Tabla 8 aparece la relación de instituciones de origen español referenciadas en las contribuciones recuperadas por ambas bases de datos. La posición que ocupan en la clasificación se establece en función del número de autores adscritos a esas instituciones cuyo número aparece referenciado entre paréntesis junto al nombre concreto de cada universidad.

Tabla 8. Instituciones de origen español recogidas del análisis de ambas bases de datos. Fuente: elaboración propia.

\begin{tabular}{|c|c|}
\hline Instituciones de origen español de la base de & Instituciones de origen español de la base de datos \\
datos Lisa & Lista \\
\hline Universidad de Barcelona (5) & Universidad de Barcelona (3) \\
Universidad Rovira i Virgili (3) & Universidad Rovira i Virgili (2) \\
Universidad de Salamanca (3) & Universidad del País Vasco (1) \\
Universidad de Granada (2) & Universidad Oberta de Catalunya (1) \\
Universidad de San Pablo CEU (2) & \\
Universidad Complutense de Madrid (1) & \\
Universidad del País Vasco (1) & \\
Universidad de Málaga (1) & \\
Universidad de Zaragoza (1) & \\
Universidad Oberta de Catalunya (1) & \\
\hline
\end{tabular}




\subsection{PROCEDENCIA GEOGRÁFICA DE LOS AUTORES DE LAS CON- TRIBUCIONES ANALIZADAS}

Determinamos la procedencia geográfica de los autores de trabajos-fuente según la ubicación de su filiación instituciones reflejada en sus contribuciones. Cuando un autor ha trabajado en más de un sitio, se ha consignado la última procedencia o aquélla en la que ha permanecido más tiempo. Además se ha consignado una única procedencia geográfica para aquellos autores con más de una contribución firmada.

El análisis comparado de resultados de ambas bases de datos nos permite afirmar que la mayoría de los autores se concentran en los Estados Unidos de América y en el Reino Unido. Reflejo característico del ámbito anglosajón en el que se enmarcan las bases de datos seleccionadas para el estudio de producción.

Además, resulta coincidente en ambas bases de datos que la ciudad de Sheffield se posicione como la ciudad que aglutina un mayor número de profesionales especializados en la materia de estudio.

En función de la clasificación de los países mejor posicionados de las bases de datos Lisa y Lista en función del criterio producción/país, España se sitúa en cuarta y séptima posición, respectivamente.

Las diferencias emergentes del análisis de resultados de las bases de datos Lisa y Lista se concentran en dos aspectos: la mayor variedad de países recogidos en la base de datos Lista y el mayor número de Comunidades Autónomas de España referenciados en la base de datos Lisa.

Por otro lado, los resultados vuelven a ser coincidentes al posicionar a Barcelona como la ciudad española más representativa por concentrar el mayor número de autores especializados en la materia de estudio. Entre ellos, el doctor Borrego Huerta vinculado a la Universidad de Barcelona que ha destacado por ser el más productivo entre los autores con procedencia institucional española en la base de datos Lisa según la materia estudiada.

\section{CONCLUSIONES}

A pesar de que hemos observado en los resultados anteriores las discrepancias en el análisis de ambas bases de datos, a posteriori muestran un alto grado de similitud de los datos obtenidos reflejándose, entre otros aspectos, en la pequeña desviación en el cómputo global de fuentes de publicación y en el número de títulos más productivos, así como, en las carencias formales en los registros de ambas bases. Las bases de datos Lisa y Lista presentan unos tesauros caracterizados por su falta de exhaustividad y, en general, necesitan una mayor normalización que se aplique a la información contenida en sus registros. Llegado a este punto, podemos afirmar que el uso conjunto y combinado de estas bases de datos es imprescindible proporcionando los datos necesarios para obtener una visión más amplia y completa del estado de la Investigación Cualitativa en nuestro ámbito. 
Existe una producción escasa de trabajos que adoptan un enfoque cualitativo en el área de Biblioteconomía y Documentación (521 trabajos-fuente) si los comparamos con el número total de registros almacenados en ambas bases de datos ${ }^{18}$. Sin embargo, el ritmo productivo de las contribuciones es regular apuntando a una tendencia ascendente.

En el año 2006 se concentra el mayor número de trabajos sobre nuestra temática de estudio en ambas bases de datos. Destacando el quinquenio comprendido entre los años 2006-2010 como el más productivo, aunándose factores de actualización y representación de la muestra de trabajos recuperados en las bases de datos.

A la luz de los datos expuestos, podemos afirmar que la lengua española ocupa posiciones superiores en relación con las otras lenguas no inglesas recogidas en los resultados de ambas bases de datos. Tanto en función de la relación producción/idioma como en la variedad idiomática de las contribuciones recuperadas en ambas bases de datos sobre nuestro tema/objeto de estudio la base de datos Lisa destaca sobre la base de datos Lista.

Las fuentes de publicación que presentan mayores tasas de producción en función del número de ítems proporcionados por las bases de datos Lisa y Lista fueron las siguientes:

a) Según los resultados de la base de datos Lisa: Library and Information Science Research.

b) Según los resultados de la base de datos Lista: Choice: Current Reviews for Academic Libraries.

Sin embargo, la publicación Journal of Documentation es la que concentra una alta tasa de ítems sobre el tema objeto de nuestra investigación en ambas bases de datos y aparece mejor posicionada en el ranking de referencia Journal Citation Reports (JCR) en la categoría temática "Information Science \& Library Science". Además, El Profesional de la Información, es el único título de origen español coincidente entre el cómputo global de fuentes de publicación de ambas bases de datos.

La procedencia geográfica de los autores de trabajos-fuente se concentra en los Estados Unidos de América, el Reino Unido y Australia en este orden, respectivamente. Aunque la ciudad de Sheffield es la que aglutina el mayor número de autores de trabajos-fuente. En España, en función de la procedencia geográfica de los autores de trabajos-fuente, la Comunidad Autónoma de Cataluña se configura como la más productiva. Atendiendo a las provincias será Barcelona la más productiva según los registros proporcionados por las bases de datos.

La Universidad de Sheffield se perfila como la institución académica más representativa aglutinando un mayor número de profesionales especializados en la materia de estudio. Los resultados de la Universidad de Sheffield se concentran en el Depar-

\footnotetext{
${ }^{18}$ Según datos oficiales de ProQuest, la frecuencia de actualización de la base de datos Lisa es de cada dos semanas agregándose más de 500 registros.
} 
tamento de Documentación, creado en 1963 se ha convertido en un "centro de gran prestigio en el ámbito académico, tanto nacional como internacional, debido principalmente al valor científico de sus investigaciones, a la calidad de la enseñanza que imparte y obviamente al renombre y experiencia cientifica del profesorado" (Muñoz de Solano y Palacios, 2003: 134). En España, la Universidad de Barcelona se perfila como centro de referencia nacional en la investigación de la materia, en concreto, su Departamento de Biblioteconomía y Documentación a cuya filiación institucional pertenecen Candela Ollé, Ángel Borrego y Mario Pérez Montoro, entre otros autores. Seguida en segunda posición por las Universidades Rovira i Virgili con los trabajos de Paul Capriotti, Gerard Ryan y Mireia Valverde y la Universidad de Salamanca con la filiación institucional de José Luis Alonso Berrocal, José Antonio Cordón García y Ángel Francisco Zazo. En tercer lugar por número de filiaciones institucionales destaca la Universidad de Granada con el trabajo de María Pinto Molina y AnneVinciane Doucet y la Universidad de San Pablo CEU con los autores Viviana Fernández Marcial y Carlos García Figuerola. Finalmente, en cuarto lugar se posicionan cinco instituciones académicas con una única filiación institucional, respectivamente. La Universidad Complutense de Madrid con Rosario Arquero Avilés, la Universidad del País Vasco con Teresa Agirreazaldegi, la Universidad de Málaga con Carmen Gómez Camarero, la Universidad de Zaragoza con Javier García Marco y la Universidad Oberta de Catalunya con Núria Ferran Ferrer.

\section{REFERENCIAS BIBLIOGRÁFICAS}

ARQUERO AVILÉS, R. "Estudios cualitativos sobre análisis y evaluación de la investigación en Biblioteconomía y Documentación". Revista bibliográfica Investigación Bibliotecológica, 16(32), 2002: 5-25.

ARQUERO AVILÉS, R. Análisis de la investigación española en Biblioteconomía y Documentación: 1975-1984, [tesis doctoral]. Madrid: Universidad Complutense de Madrid, 2001.

BALBOA, A. C "El incidente crítico como técnica para recolectar datos". Métodos Cualitativos para estudiar a los usuarios de la información. México: UNAM, Centro Universitario de Investigaciones Bibliotecológicas, 2008.

BECK, S. E. Y MANUEL, K. Practical research methods for librarians and information professionals. New York [etc.]: Neal-Schuman, 2008.

BOOTH, A. The Qualitative Evidence Base for Library and Information Practice: how much? How good? How useful?, [en línea], 2009. $<$ http://blogs.kib.ki.se/eblip5/presentations/AndrewBoothpresentation.pdf.>. [Consulta: 17-07-2012].

BORREGO HUERTA. A. Metodología cualitativa de investigación en Biblioteconomía y Documentación: 1993-1999, [tesis doctoral]. Salamanca: Universidad de Salamanca, 2001. 
BORREGO HUERTA. A . "La investigación cualitativa y sus aplicaciones en Biblioteconomía y Documentación”. Revista Española de documentación científica, 1999, 22(2), 139-156.

BRUYN, S. "The methodology of participant observation". Reader in research methods for librarianship. Washington, DC: Microcard Editions, 1970: 172-185.

COLLER, X. Estudio de casos. Madrid: CIS, Centro de Investigaciones Sociológicas, 2000.

CÓRDOBA, S. "Los métodos cualitativos en los estudios de usuarios: Una revisión bibliográfica".Forinf@ Online: Revista Iberoamericana sobre Usuarios de la Información, 1998: 5-8.

DELGADO LÓPEZ-COZAR, E.”¿Por qué enseñar métodos de investigación en las facultades de Biblioteconomía y Documentación?”. Anales de Documentación, 4, 2001:51-71.

FERNÁNDEZ HERNÁNDEZ, S. y RIVERA, Z "El paradigma cualitativo y su presencia en las investigaciones de la Bibliotecología y la Ciencia de la Información". ACIMED [online], 20 (3), 2009:6-30.

FIDEL, R. "Are we there yet? : Mixed methods research in library and information science". Library \& Information Science Research, 30 (4), 2008:265-272.

FRÍAS, J. A. y BORREGO, A. "Más allá de la cantidad: la incorporación de los métodos cualitativos a la investigación en Información y Documentación". Metodologías de Investigación en Información y Documentación. Salamanca: Universidad de Salamanca, 2004:193-211.

FRÍAS MONTOYA, J. A Seminario de Técnicas cualitativas de investigación en Ciencias de la Información, [en línea]. Blogtecaria, 2010. $<$ http://blogtecaria.blogspot.com.es/2010/09/incentivo-la-investigacioncualitativa.html >. [Consulta: 15-06-2012].

GLITZ, B. "The focus group technique in library research: An introduction". Bulletin of the Medical Library Association, 85(4), 1997:385-390.

GONZÁLEZ TERUEL, A. Los estudios de necesidades y usos de la información: fundamentos y perspectivas actuales. Gijón: Trea, 2005.

GOODMAN, V. D. Qualitative research and the modern library. Oxford: Chandos, 2011.

HASSAN-MONTERO, Y. y ORTEGA-SANTAMARÍA, S. Informe APEI sobre usabilidad. Gijón: APEI, Asociación Profesional de Especialistas en Información, 2009.

HERNÁNDEZ SALAZAR, P. "La importancia de la satisfacción del usuario". Documentación de las Ciencias de la Información 34, 2011:349-368.

HERNÁNDEZ SALAZAR, P. "Utilización de métodos cualitativos para realizar estudios de usuarios". Métodos cualitativos para estudiar a los usuarios de la información. México: UNAM, Centro Universitario de Investigaciones Bibliotecológicas, 2008. 
LAWAL, I. O. Library and information science research in the 21st century: A guide for practising librarians and students. Oxford [etc.]: Chandos, 2009.

MARTÍN MORENO, C. "Metodología de investigación en estudios de usuarios". Revista General de Información y Documentación 17(2), 2007:29-149.

MATTHEWS, J. R. The Evaluation and Measurement of Library Services. Westport: Libraries Unlimited, 2007.

MELLON, C. A. Naturalistic Inquiry for Library Science: Methods and Applications for Research, Evaluation, and Teaching. New York: Greenwood Press, 1990.

MORENA DE DIAGO, B. Análisis de la Investigación Cualitativa en el área de Biblioteconomía y Documentación: 1981-2010, [tesis doctoral]. Madrid: Universidad Complutense, 2012.

MUELA-MEZA, Z. M. "Una introducción a las metodologías de investigación cualitativa aplicadas a la bibliotecología". BiblioDocencia: Revista de Profesores de Bibliotecología 2 (12), 2006:4-12.

MUÑOZ de SOLANO y PALACIOS, B. "Los estudios de Biblioteconomía y Documentación en el Reino Unido. Un caso específico: la universidad de Sheffield". Revista General de Información y Documentación, 13(1), 2003:133-150.

MURUA ANZOLA, I. "Internet, campo y herramienta de investigación". Revista Electrónica Teoría de la Educación: Educación y Cultura en la Sociedad de la Información 8(1), 2007:28-41.

MYERS, M. D. y NEWMAN, M. "The qualitative interview in IS research: Examining the craft". Information \& Organization, 17(1), 2007:2-26.

OLLÉ, C. y BORREGO, A. "Librarians' perceptions on the use of electronic resources at Catalan academic libraries: Results of a focus group". New Library World 111 (1/2), 2010:46-54.

PATTON, M. Q. Qualitative evaluation and research methods. Newbury Park: SAGE, 1990.

PÉREZ-MONTORO GUTIÉRREZ, M. Arquitectura de la información en entornos web. Gijón: Trea, 2010.

POWELL, R. R., CONNAWAY, L.S. Basic research methods for librarians. Westport, Connecticu [etc.]: Libraries Unlimited, 2004.

RIAZA CHAPARRO, M. Nuevas técnicas de estudios de usuario aplicadas a bibliotecas. Revista General de Información y Documentación. 17 (2), 2007:45-61

RUIZ ABELLÁN; J., LÓPEZ-HUERTAS PÉREZ, M. J. "Técnicas cualitativas para la representación del conocimiento de usuarios en un sistema de información especializado". La representación y la organización del conocimiento en sus distintas perspectivas: su influencia en la recuperación de la información: actas del IV Congreso ISKO-España EOCONSID'99, 22-24 de abril de 1999. Granada: Sociedad Internacional para la Organización del Conocimiento, 1999:435-441.

SANZ CASADO, E. Manual de estudios de usuarios. Madrid: Fundación Germán Sánchez Ruipérez: Pirámide, 1994. 
TAYLOR, S. J. y BOGDAN, R. Introducción a los métodos cualitativos de investigación: la búsqueda de significados. Barcelona [etc.]: Paidó, 1994s.

WILSON, T. "Information science" and research methods, [en línea], 2002. $<$ http://informationr.net/tdw/publ/papers/slovak02.html $>$. [Consulta: 19-11-2011]. 


\section{ANEXO: Propuesta de sistematización}

\section{Técnicas conversacionales}

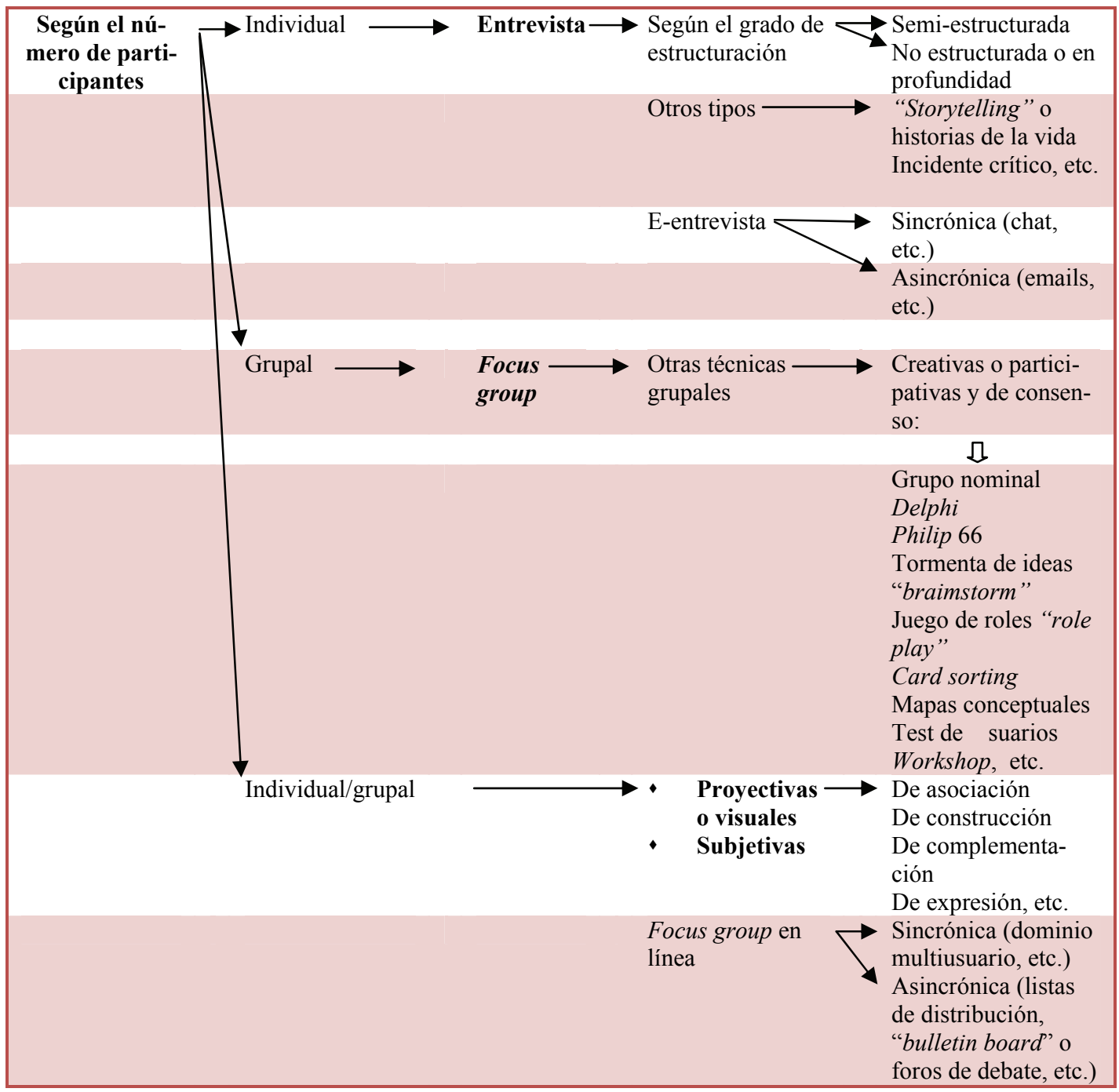




\section{Técnicas documentales}

\begin{tabular}{|ll|}
\hline $\begin{array}{l}\text { Según la finalidad del ente } \\
\text { productor }\end{array}$ & Documentos ajenos a la investigación (públicos o privados) \\
\hline Según la información registrada $\longrightarrow$ & Documentos elaborados en el curso de la investigación \\
\hline Tipos más comunes $\longrightarrow$ Documentos multimedia (animaciones, vídeos, hipervínculos, \\
etc.)
\end{tabular}

$\underline{3 \text { Técnicas observacionales }}$

\begin{tabular}{|c|c|c|}
\hline \multirow[t]{3}{*}{$\begin{array}{l}\text { Según la fun- } \\
\text { ción activa }\end{array}$} & \multicolumn{2}{|l|}{$\longrightarrow$ Del usuario $\longrightarrow$ Protocolos verbales } \\
\hline & $\begin{array}{l}\text { Del investi- } \\
\text { gador }\end{array}$ & Cliente oculto o "mystery shopping" \\
\hline & \multicolumn{2}{|c|}{$\triangle$ Como investigador $\longrightarrow$ Observación } \\
\hline & & $\mathbb{Z}$ \\
\hline $\begin{array}{l}\text { Observación en } \\
\text { línea }\end{array}$ & & $\begin{array}{l}\text { Según el grado de participación del } \\
\text { observador }\end{array}$ \\
\hline I & & I \\
\hline Sincrónica & $\longrightarrow$ Netnografía & Observación \\
\hline Asincrónica & $\longrightarrow \begin{array}{l}\text { Etnografía } \\
\text { virtual }\end{array}$ & $\begin{array}{l}\text { Observación participante (activa, } \\
\text { pasiva) }\end{array}$ \\
\hline
\end{tabular}

